\title{
Development of Fast-Neutron Directional Detector for Fusion Neutron Profile Monitor at LHD ${ }^{*}$
}

\author{
Eiji TAKADA, Akihiro FUJISAKI, Naoki NAKADA, Mitsutaka ISOBE ${ }^{1,2)}$, Kunihiro OGAWA ${ }^{1,2)}$, \\ Takeo NISHITANI ${ }^{1)}$ and Hideki TOMITA ${ }^{3)}$ \\ National Institute of Technology, Toyama College, Toyama 939-8630, Japan \\ ${ }^{1)}$ National Institute for Fusion Science, Toki 509-5292, Japan \\ ${ }^{2}$ SOKENDAI (The Graduate University for Advanced Studies), Toki 509-5292, Japan \\ ${ }^{3)}$ Nagoya University, Nagoya 464-8603, Japan
}

(Received 22 November 2015 / Accepted 23 January 2016)

\begin{abstract}
Fast-neutron directional detectors using scintillating optical fibers have been adopted for neutron emission profile measurement in magnetic confinement fusion devices. Because of their benefits of rapid response and directional properties to reduce restrictions on shielding, they are anticipated for installation in the Large Helical Device (LHD) of the National Institute for Fusion Science (NIFS). As described herein, the system design has been re-examined based on the results of recent experiments and simulations. Results show that fast neutrons with high energy of $14 \mathrm{MeV}$ are attenuated by a self-shielding effect of scintillating fiber. Moreover, it has been demonstrated that the scintillation photons are attenuated in several centimeters before reaching the photon detector. The control of the photon transmission efficiency is important to enhance the directional property of the system. Along with reduced effects of the background gamma rays, adopting a thin scintillating fiber is effective. Further evaluation should be conducted to design a detector system considering the detecting efficiency, directional property, and gamma ray effect for application to an actual radiation field of the LHD.
\end{abstract}

(c) 2016 The Japan Society of Plasma Science and Nuclear Fusion Research

Keywords: neutron measurement, fast neutron directional detector, scintillating optical fiber, large helical device (LHD), design

DOI: $10.1585 /$ prr.11.2405020

\section{Introduction}

Energetic particle confinement performance has been assessed through neutron emission profile measurements taken in magnetically confined experimental fusion devices. Scintillation detectors with multichannel collimators have been installed in many experimental fusion devices such as the Tokamak Fusion Test Reactor [1], the Joint European Torus [2], and JT-60 Upgrade [3]. One system comprises scintillating optical fiber, which can operate as a directional fast neutron detector. The system has been expected to reduce requirements for collimation performance. At the Large Helical Device (LHD) of National Institute for Fusion Science (NIFS), operation with deuterium fuel is scheduled to be conducted in several years. The scintillating fiber-based system is being planned for installation as a neutron profile monitor for those experiments on the basement level of the LHD [4] (Fig. 1). The scintillating fiber-based system was developed more than 20 years ago, where the scintillating fiber diameter was $1 \mathrm{~mm}$ and its length was $10 \mathrm{~cm}[5,6]$. Since then, the system design has not been reconsidered. In this study, the authors evaluated the effects of the design parameters

author'se-mail: takada@nc-toyama.ac.jp

*) This article is based on the presentation at the 25th International Toki Conference (ITC25).

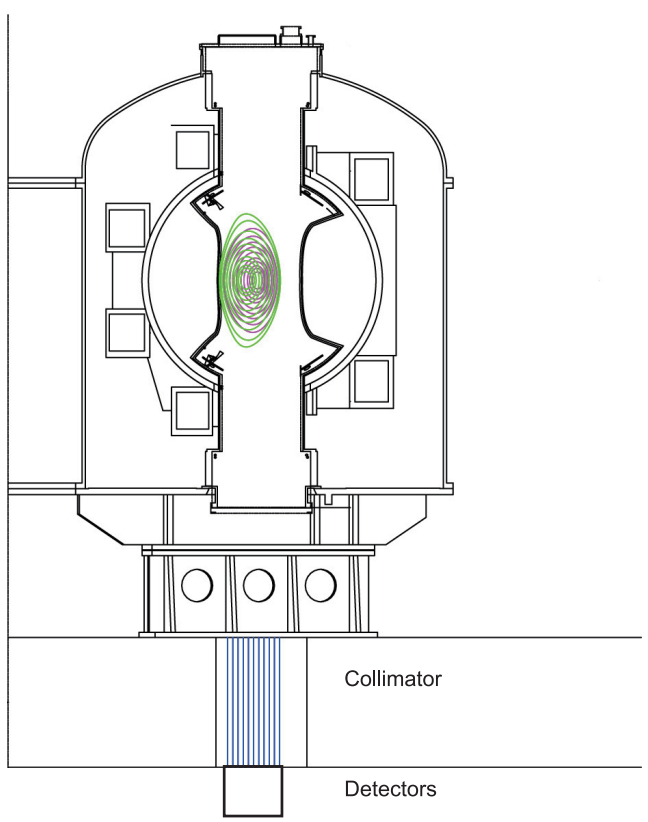

Fig. 1 Schematic view of the neutron profile monitor for LHD [1].

and tried to optimize it for $14 \mathrm{MeV}$ neutron measurement. From the study, self-shielding of $14 \mathrm{MeV}$ neutron by the scintillating fiber itself was revealed as a source of degra- 


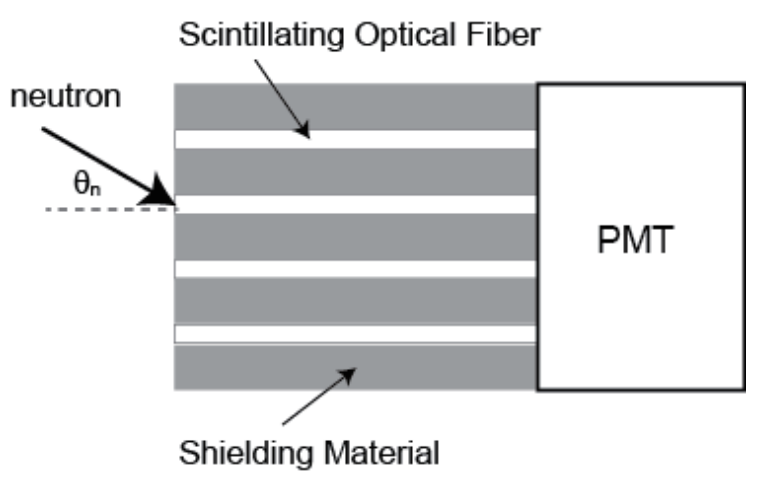

Fig. 2 Structure of fast neutron directional detector.

dation of angular resolution of the system. Some scintillation photons were regarded as transmitted and reflected at the walls of the holes into which the scintillating fibers were inserted. Because of this phenomenon, the number of the photons decreases with longer transmitting distance. Considering these effects, the system design has been reconsidered for LHD deuterium experiments.

\section{Fast Neutron Directional Detector Based on Scintillating Fiber}

The system comprises several scintillating fibers inserted in shielding material, by which recoiled protons are prevented from entering another scintillating fiber. The scintillating fibers are connected to a photomultiplier tube (PMT) to convert scintillation photons into electrical signals. Because the recoil protons are generated to a forward direction with greater probability, the energy deposition is greater when the neutrons are incident with smaller value of $\theta_{\mathrm{n}}$ in Fig. 2, which results in a larger output pulse from the PMT. Therefore, by setting a proper discrimination level for the output pulse height, events with small $\theta_{\mathrm{n}}$ can be measured selectively. In the system installed in JT$60 \mathrm{U}$, the diameter of the scintillating fiber was $1 \mathrm{~mm}$. Its length was $10 \mathrm{~cm}[5,6]$.

\section{Experimental and Simulation}

To evaluate the properties of the previously developed measurement system, we conducted simulations with the Monte Carlo Simulation Code PHITS [7]. Experiments were conducted at the Fast Neutronics Source (FNS) of the Japan Atomic Energy Agency (JAEA) [8,9]. Figure 3 shows the simulated energy deposition inside the scintillating fiber with neutron incident angles of $0 \mathrm{deg}$. and 70 deg. Figure 4 shows measured pulse height distributions at equal incident angles. Results are shown for scintillating fibers with lengths of $10 \mathrm{~cm}$.

In the simulated and experimentally obtained results, one can observe the edge at the highest energy region. However, the edge shapes in the experiments were broader than those in the simulation. In Fig. 5, for $5 \mathrm{~cm}$-long scintillating fibers, the relation between the neutron incident

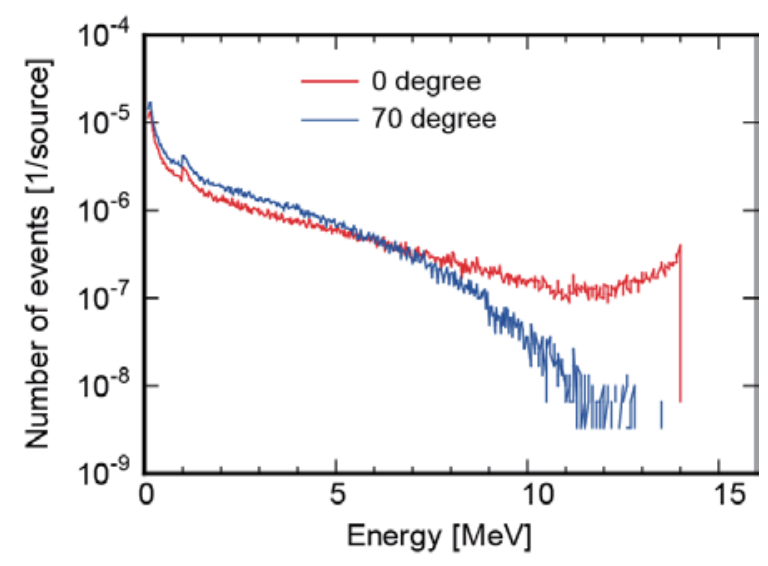

Fig. 3 Energy deposition inside the scintillating fiber simulated by PHITS.

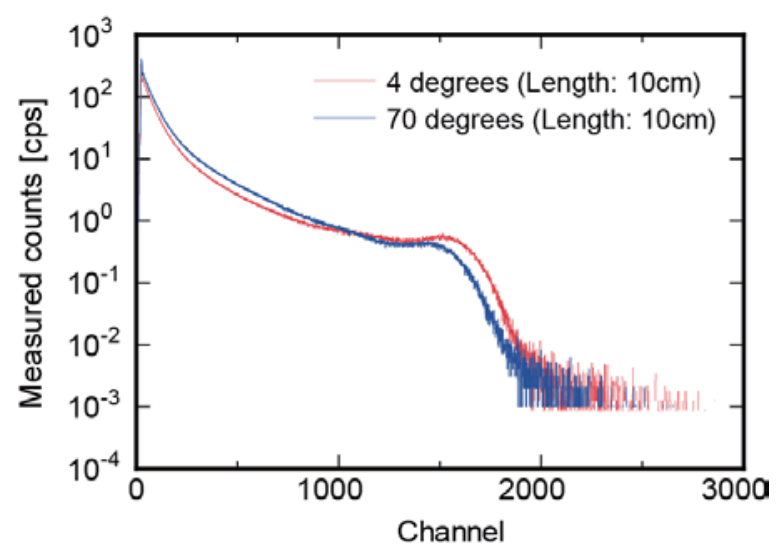

Fig. 4 Measured pulse height distributions by scintillating fiber detectors, where the diameter was set to $1 \mathrm{~mm}$ and the lengths were $10 \mathrm{~cm}$.

angle and the numbers of events with energy deposition of more than $13 \mathrm{MeV}$ on the neutron incident angle is shown. In the same figure, counts measured at the edges are also shown. Both the simulated and measured numbers were normalized by those with the incident angle of $0 \mathrm{deg}$. It is apparent that the directional property is worse in the experiments than in the simulation.

\section{Discussion and Detector Redesign}

The differences between simulation and experiment probably resulted from the following effects: (1) gamma rays in the experimental room, (2) self-shielding of the neutrons by the scintillating fiber itself, and (3) decrease of the scintillation photons while they transmit through the scintillating fiber. We conducted additional simulations to ascertain ways to reduce these effects.

(1) Effects of background gamma rays

Simulations with secondary gamma rays revealed no significant effect on the results. Therefore, the background 


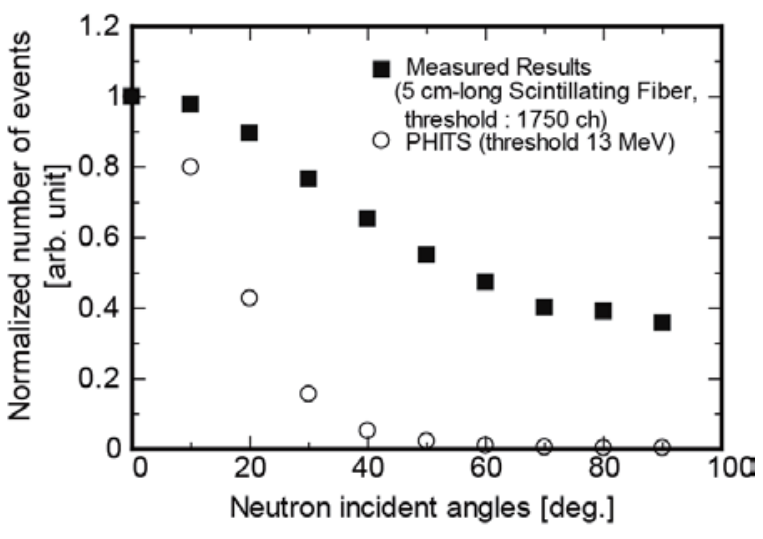

Fig. 5 Angular distribution of the events by simulations and experiments, where the values have been normalized to those with the neutron incident angle of $0 \mathrm{deg}$.

gamma rays in the experimental room are expected to have been a main factor affecting the pulse height distribution. More scintillation photons are known to be generated in the interaction with electrons than with protons. By the resultant phenomenon, the signal pulse height generated by a gamma ray will be greater than that generated by a neutron having the same energy [10].

To find some means to reduce the gamma ray effect, we conducted simulations for the scintillating fiber with 0.5 -mm-diameter. In the simulations, the energies of gamma rays and neutrons were set to $14 \mathrm{MeV}$. We recognize that the energy of $14 \mathrm{MeV}$ for gamma rays is unrealistic in the fusion experimental environment. However, we used the value because the energy distribution of gamma rays in LHD is unknown. For a 0.5-mm-diameter scintillating fiber, for $14 \mathrm{MeV}$ gamma rays, the calculated number of events with energy deposition of more than $4 \mathrm{MeV}$ becomes 0.036 times smaller than that for 1-mm-diameter scintillating fiber, whereas the ratio is 0.086 for $14 \mathrm{MeV}$ neutrons. These ratios are less than 0.25 , which is calculated from the change of the sensing volume. That is true because we set the energy thresholds, as described in section 3 , to clarify the proportional property. In the case of thin fiber, to generate pulses over the threshold, the permissible solid angle becomes smaller, which causes the lower efficiencies. Moreover, because the stopping power of the fiber is lower for electrons than for protons, the restriction for the solid angle for electrons is stricter than that for protons, which is the cause of the lower sensing efficiency for gammas. These results suggest that adopting 0.5 -mm-diameter scintillating fiber can reduce the background gamma ray effects.

(2) Self-shielding of the neutrons by the scintillating fiber itself

To evaluate the self-shielding effect of neutrons by the scintillating fiber, PHITS simulation was conducted for the setup depicted in Fig. 6 . In the $14 \mathrm{MeV}$ neutron beams

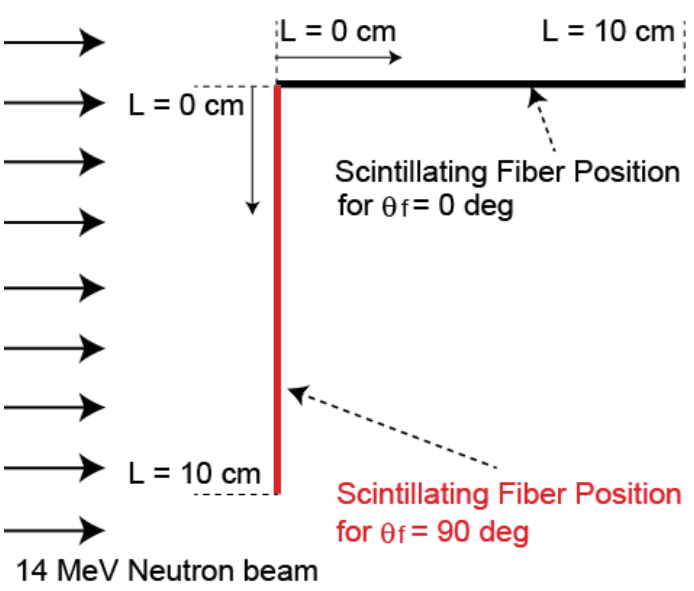

Fig. 6 Setup for the PHITS simulation to evaluate the selfshielding effect by the scintillating optical fiber.

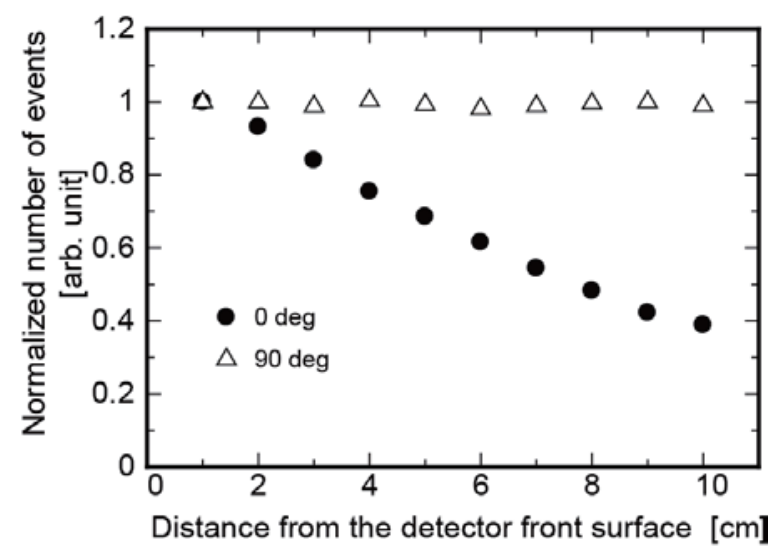

Fig. 7 Calculated distribution of the recoiled protons in a scintillating fiber, where $14 \mathrm{MeV}$ neutrons enter into it as shown in Fig. 6.

with $12 \mathrm{~cm}$ width, a scintillating optical fiber was set at two angles: $\theta_{\mathrm{f}}=0 \mathrm{deg}$. and $90 \mathrm{deg}$. Figure 7 shows the distribution of the recoiled protons in a scintillating fiber. Apparently, when $\theta_{\mathrm{f}}=0 \mathrm{deg}$, the number of recoil protons decreases as the depth from the neutron incident position increases. Up to $3 \mathrm{~cm}$ depth, the number decreases to 0.8 times of the value at the surface. At the deeper position, it decreases gradually and becomes almost 0.4 times at $10 \mathrm{~cm}$ depth. Therefore, the scintillation photons are generated mainly at a position far from the PMT.

(3) Decrease of the scintillation photons while they transmit through scintillating fiber

In the measurement system, scintillating fibers were input into the holes made through the shielding material as aluminum. As presented in Fig. 8, to evaluate the decrease of the scintillation photons in transmission process, experiments were conducted using a ${ }^{137} \mathrm{Cs}$ gamma ray source by changing the gamma ray incident position on the scintillating fiber. In the experiments, scintillating fibers with 1$\mathrm{mm}$-diameter and $10 \mathrm{~cm}$ length were used. Figure 9 shows 


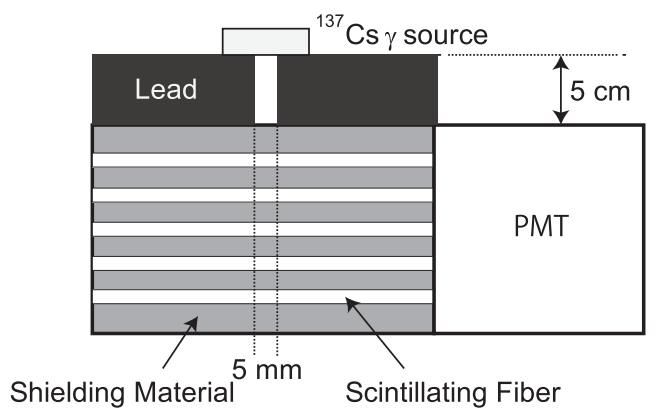

Fig. 8 Experimental setup to evaluate the attenuation of photons with changing the gamma ray incident position.

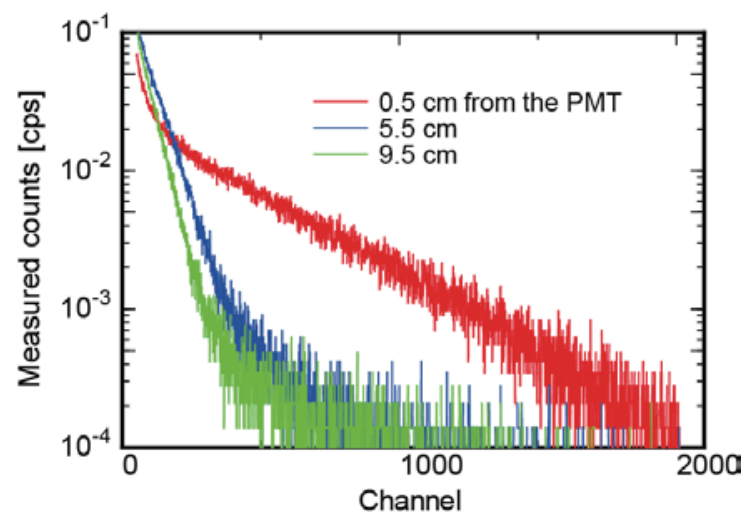

(a) Without an AR layer

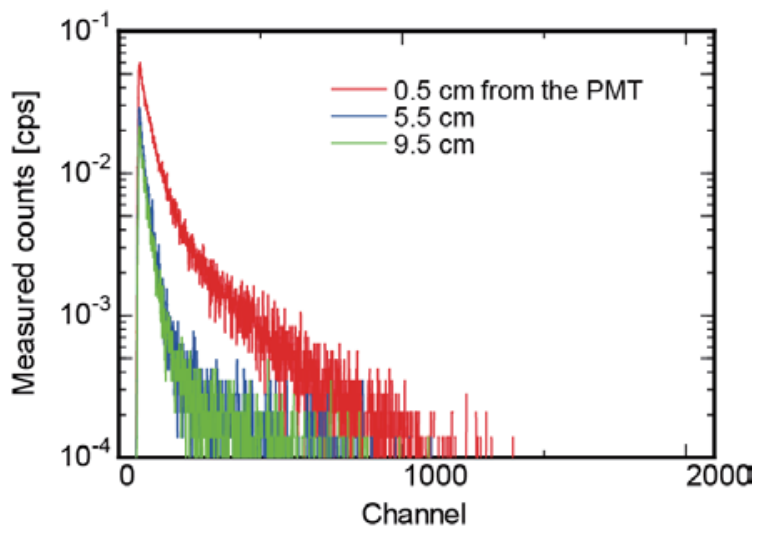

(b) With an AR layer

Fig. 9 Measured pulse height distributions when ${ }^{137}$ Cs gamma rays were irradiated on to some points along the scintillating fiber.

the measured pulse height distributions for incident positions of $0.5 \mathrm{~cm}, 5.5 \mathrm{~cm}$, and $9.5 \mathrm{~cm}$ from the PMT. In Fig. 9 (a), the results without an anti-reflection (AR) layer on the scintillating fiber surface are shown, whereas the results in Fig. 9(b) are results with the AR layer. Figure 9 clarifies that, when without the AR layer, the counts decreased extremely rapidly with increasing distance. However, with the AR layer, although the counts are small at all positions, the change of the distribution is small. In Fig. 10, the measured counts over $500 \mathrm{ch}$ are depicted, where the

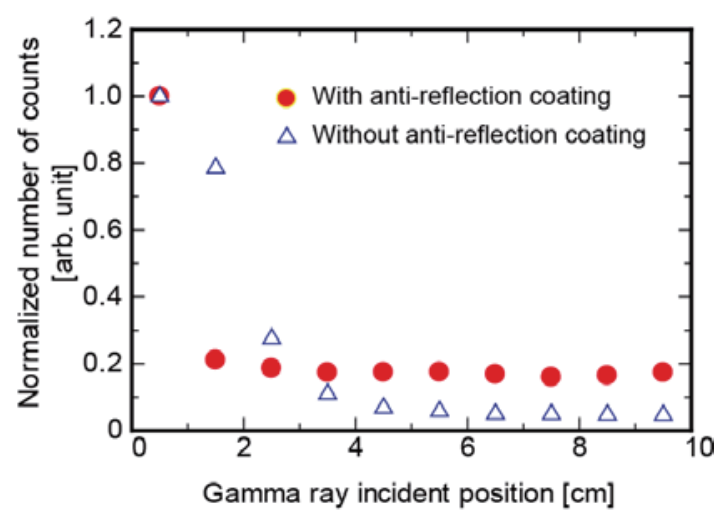

Fig. 10 Measured counts over a threshold $(500 \mathrm{ch})$ with change of the gamma ray incident position. Data were normalized to the value at the distance of $0.5 \mathrm{~cm}$.

counts have been normalized by the value at a distance of $0.5 \mathrm{~cm}$. We conclude that attenuation of the scintillation photons has been suppressed by the AR layer.

To elucidate the transmission process, the scintillation photons must be divided into two groups: (1) photons emitted to the directions which meet the total reflection condition of the scintillating fiber and (2) photons which do not completely reflect at the core/cladding interface and which are reflected at the wall boundary of the holes in which the scintillating fibers are set. Our experiments show that the photons of the latter group decreased rapidly in the first $3 \mathrm{~cm}$. Subsequently, they become almost constant. The change of the collection efficiency affects the pulse height of the output which degrades the directional property. When preparing the AR layer on the scintillating fiber surface, the photons in group (2) decreased more rapidly before becoming constant.

From these results, the following modification of parameters is expected to enhance the directional property of the detector. First, the scintillating fiber diameter must be less than $1 \mathrm{~mm}$ to reduce the gamma ray effect. Regarding the length, the results implied that, two methods are possible to maintain the collection efficiency of the photons as uniform as possible: (A) increasing the reflection efficiency of the wall surface to the greatest extent possible and setting the scintillating fiber length as short as possible, less than $1.5 \mathrm{~cm}$, or (B) preparing an AR layer on the scintillating fiber surface and setting their length as longer, more than $3 \mathrm{~cm}$. In case (a), we use all the scintillation photons. In case (b), only the photons which meet the total reflection condition of the scintillating fiber is used. Smaller diameter and shorter length are expected to decrease the measuring efficiency of the system. Therefore, it will be necessary to increase the number of scintillating fibers. Additional evaluation is expected to be necessary to design the detector system considering the detection efficiency, directional property, and gamma ray effect to apply it to the real radiation field of the LHD. 


\section{Summary}

We have studied the design parameters which determine the properties of scintillating fiber-based fast neutron detector that was developed and installed in some fusion experimental devices. In redesigning the parameters, we must consider self-shielding of the neutrons by the scintillating fiber itself. Moreover, the wall condition of the holes into which the scintillating fibers are inserted is extremely important because the scintillation photons are attenuated by being reflected there. The strategy of setting an AR layer outside the scintillating fiber or not engenders two designs: short length without an AR layer or long length with an AR layer. The gamma ray effect on the angular resolution of the system can also be reduced by selecting a smaller scintillating fiber diameter. Considering these present results, the actual design of the total detection sys- tem should be determined based on the estimated neutron and gamma ray fluxes at the detector position in the LHD.

[1] A.L. Roquemore et al., Rev. Sci. Instrum. 61, 3163 (1990).

[2] L. Bertalot et al., Proc. of the 23rd Symposium of Fusion Technology 74 (1-4), 835-839 (2005).

[3] M. Ishikawa et al., Rev. Sci. Instrum. 73, 4237 (2002).

[4] K. Ogawa et al., Rev. Sci. Instrum. 85, $11 \mathrm{E} 110$ (2014).

[5] G.A. Wurden et al., Rev. Sci. Instrum. 66 (1), 901 (1994).

[6] H. Harano, JAERI-Research 97-060 (1997) (in Japanese).

[7] T. Satoet al., J. Nucl. Sci. Technol. 50 (9), 913 (2013).

[8] Y. Ikeda et al., JAERI Report 1312 (1988).

[9] C. Konno et al., JAERI Report 1329 (1993).

[10] G.F. Knoll, Radiation Detection and Measurement, Third Edition, John Wiley \& Sons, 2000, ISBN: 9780471073383. 